\title{
REVERSAL OF IATROGENIC MYOCARDIAL EDEMA AND RELATED ABNORMALITIES OF DIASTOLIC PROPERTIES IN THE PIG LEFT VENTRICLE
}

David A. Dean, MD ${ }^{\text {** }}$

Mehrdad M. R. Amirhamzeh, MD ${ }^{a}$

Chao-Xiang Jia, MD ${ }^{\mathrm{a}}$

Santos E. Cabreriza, $\mathrm{MBA}^{\mathrm{a}}$

David G. Rabkin, $\mathrm{BA}^{\mathrm{a}}$

Robert Sciacca, EngScD ${ }^{\text {b }}$

Marc L. Dickstein, $\mathrm{MD}^{\mathrm{c}}$

Henry M. Spotnitz, MD ${ }^{\mathrm{a} * *}$
Objective: This study examines the resolution of iatrogenic edema and related changes in systolic and diastolic properties in the intact pig left ventricle. Methods: The coronary arteries were perfused for 50 to 60 seconds with diluted blood (hematocrit value $10 \% \pm 1 \%$, edema group, $n=5$ ) or whole blood (hematocrit value $28 \% \pm 1 \%$, control group, $n=6$ ) infused into the aortic root during aortic crossclamping in conditioned, anesthetized pigs. After whole blood reperfusion, preload reduction by vena caval occlusion was used to define systolic and diastolic properties at 15-minute intervals. Left ventricular pressure and conductance, aortic flow, and two-dimensional echocardiography were recorded. Results: Left ventricular mass (wall volume) in the edema group increased significantly compared with that in control pigs after crossclamp removal. Mass returned to preperfusion levels after 45 minutes. The ventricular stiffness constant $(\beta)$ increased significantly in the edema group versus the control group, returning to baseline by 30 minutes. The diastolic relaxation constant $(\tau)$ and base constant $(\alpha)$ did not differ between groups. There was no significant change in contractility. Conclusion: Increases in left ventricular mass and diastolic stiffness induced by coronary perfusion with hemodiluted blood resolve after $\mathbf{4 5}$ minutes of whole blood perfusion in pigs. This study defines physiologic effects of edema in the normal heart while eliminating most common confounding experimental errors. (J Thorac Cardiovasc Surg 1998;115:1209-14)
A nalysis of the effects of cardiac surgery on left ventricular (LV) systolic and diastolic properties can be nullified by myocardial edema. Edema, usually caused by ischemic injury ${ }^{1}$ or hemodilution, ${ }^{2}$ or both, is identified by increases in myocardial water content, LV weight, and echocardiographic LV

From the Departments of Surgery, ${ }^{\mathrm{a}}$ Medicine, ${ }^{\mathrm{b}}$ and Anesthesiology, ${ }^{\mathrm{c}}$ Columbia University, College of Physicians and Surgeons, New York, N.Y.

Supported in part by U.S. Public Health Service grant 1 RO1 HL-48109. Dr. Dickstein is supported by National Institutes of Health NRSA training grant HL09325-01.

Received for publication July 28, 1997; revisions requested Oct. 6, 1997; revisions received Dec. 24, 1997; accepted for publication Dec. 29, 1997.

Address for reprints: Henry M. Spotnitz, MD, Department of Surgery, Columbia University, College of Physicians and Surgeons, 622 West 168th St., PH 1422, New York, NY 10032.

*Current address: Dartmouth-Hitchcock Medical Center, Lebanon, N.H.

**George H. Humphreys II Professor of Surgery.

Copyright (C) 1998 by Mosby, Inc.

$0022-5223 / 98 \$ 5.00+0 \quad \mathbf{1 2 / 1 / 8 8 6 8 6}$ mass (wall volume). A hallmark of edema is increased LV diastolic stiffness. $^{2}$

Edema resulting from hemodilution is believed to be short-lived, fully and easily reversible. Characterization of reversible edema is needed to differentiate it from longer lasting ventricular injury. This task has been difficult and has attracted many investigators. $^{4,3-10}$ Ideally, edema should be induced and reversed without cardiac injury. Techniques that are highly likely to cause edema, including cardiopulmonary bypass (CPB) and Langendorff perfusion, should be avoided.

Pursuing this, our laboratory induced edema in the intact circulation of anesthetized rats by rapid proximal crystalloid perfusion of the distally clamped aorta, discarding right atrial drainage. On the basis of myocardial water content, edema was found to resolve after 15 minutes of whole blood reperfusion. ${ }^{11}$ Each time point required that a separate animal be put to death.

A similar study in pigs characterized induction and reversal of edema in the same animal. Abnormalities of LV stiffness and LV mass resolved after 


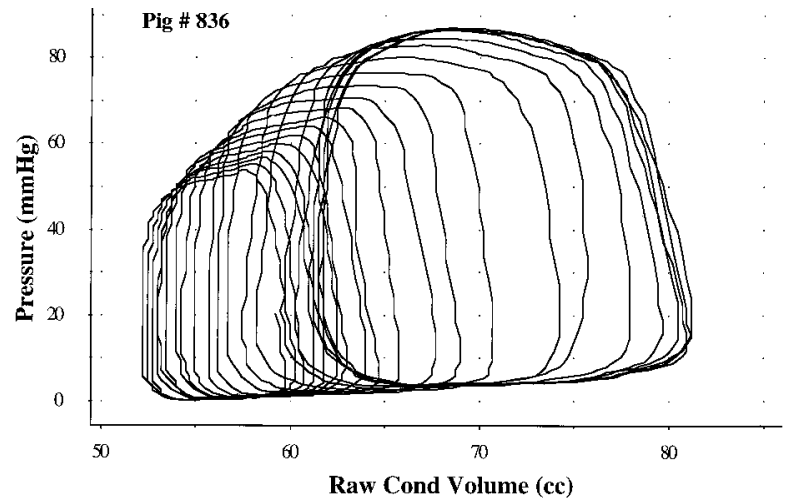

Fig. 1. Representative pressure-volume data during preload reduction by inferior vena caval occlusion. Raw conductance was converted to absolute volume by means of calibration routines described in the text.

45 minutes of whole blood reperfusion. ${ }^{12}$ Methodologic problems included myocardial ischemia and a transient need for mechanical assistance. In the present study, we sought an improved model in which the functional effects of myocardial edema alone could be directly studied in vivo for the first time.

\section{Material and methods}

Experimental protocol. Animal care complied with the "Principles of Laboratory Animal Care" formulated by the Institute of Laboratory Animal Resources, "Guide for the Care and Use of Laboratory Animals" (NIH Publication No. 86-23, revised 1985), and the position of the American Heart Association on Research Animal Use.

Conditioned Hampshire pigs ( 40 to $50 \mathrm{~kg}$ ), divided into edema $(n=5)$ and control groups $(n=6)$, were anesthetized, heparinized, and instrumented as previously described. ${ }^{12}$ The left hemiazygos vein was ligated. A $7 F$ conductance-micromanometer pressure catheter (Sentron, Inc., Federal Way, Wash.) was placed in the LV through the apex. A pulmonary artery thermistor (Baxter Healthcare Corp., Irvine, Calif.) allowed determination of cardiac output.

Intravenous fluids ( $0.9 \%$ sodium chloride) were administered at a rate of $5 \mathrm{ml} / \mathrm{kg}$ per hour. Hematocrit value and blood resistivity (which decreases with hemodilution) were not significantly different between groups at four time points during the experiment. Digitized data and two-dimensional echocardiography (2-DE) were recorded with the lungs deflated during preload reduction by vena caval occlusion as previously described. ${ }^{12}$

The coronary perfusate consisted of $167 \mathrm{ml}$ of heparinized blood in $333 \mathrm{ml}$ of Ringer's lactate solution (274 mOsm, hematocrit value $10 \% \pm 1 \%$ ) in the edema group and $500 \mathrm{ml}$ of whole blood (hematocrit value $28 \% \pm 1 \%$ ) in the control group. Normal saline solution was used for blood replacement milliliter for milliliter in each animal. An aortic cardioplegia needle and cannulas to vent the right and left atria were introduced as previously de-

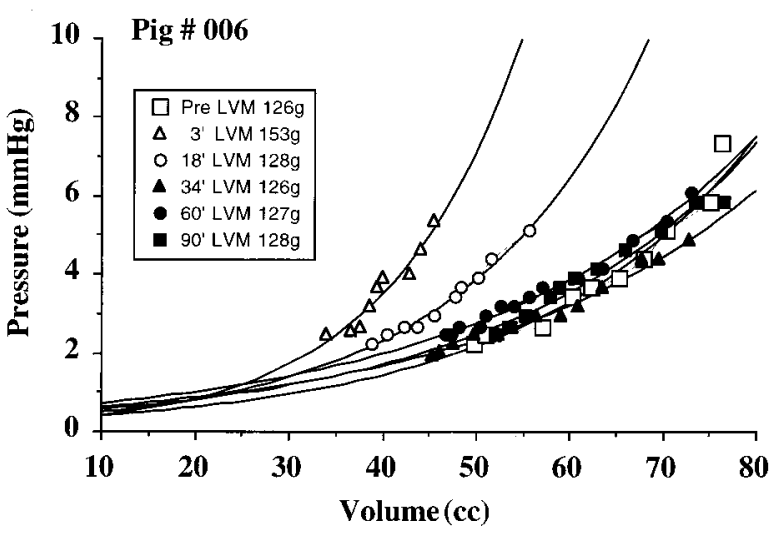

Fig. 2. Representative LV pressure-volume data after infusion of hemodiluted blood, analyzed by exponential curve fitting. The curves are shifted upward and to the left 3 and 18 minutes after reperfusion. $L V M$, LV mass by $2-\mathrm{DE}$.

scribed. ${ }^{12}$ The heart was perfused as follows: (1) The venae cavae and the main pulmonary artery were snared; (2) the aorta was crossclamped; (3) the atria were vented; (4) $500 \mathrm{ml}$ of group-specific normothermic aortic perfusate was injected at a rate of 50 to $60 \mathrm{~mm} \mathrm{Hg}$. Crossclamp time averaged less than 1 minute (edema group $57 \pm 4$ seconds, control group $51 \pm 4$ seconds). During infusion, the heart was manually compressed to avoid distention. All hearts maintained organized rhythm during infusion.

The snares and aortic clamp were then released and the vents were clamped. Epinephrine was infused $(0.05 \mu \mathrm{g} / \mathrm{kg}$ per minute) for 2 to 3 minutes until systolic blood pressure was greater than $60 \mathrm{~mm} \mathrm{Hg}$ and heart rate was less than 120 beats/min. Manual massage and circulatory support were not used.

As previously described, ${ }^{12}$ hemodynamics and 2-DE were recorded every 15 minutes for 90 minutes in the steady state and during vena caval occlusion. The heart was then arrested with potassium chloride solution. The LV was trimmed and weighed, and myocardial water content was determined.

Conductance. As previously described, ${ }^{12}$ arterial blood was collected to measure resistivity with the Leycom Sigma-5 conductance module (Rijnsburg, The Netherlands). Parallel conductance was measured before each data acquisition with injection of hypertonic saline solution, ${ }^{13}$ and $2-\mathrm{DE}$ was recorded with a $5.0 \mathrm{MHz}$ transducer, including multiple views previously described. ${ }^{12} \mathrm{LV}$ pressure and conductance were displayed with $2-\mathrm{DE},{ }^{14}$ allowing correlation at end-diastole. Alpha $(\alpha)$, the dimensionless calibration factor that converts raw conductance volume to absolute volume, was determined for each data set by comparing conductance-derived cardiac output with thermodilution and flow probe stroke volume.

Data analysis. "Pre" refers to data before crossclamping. Postreperfusion points were " 15 minutes," 3 to 15 minutes after reperfusion, "30 minutes," 16 to 30 minutes after reperfusion, " 45 minutes," 31 to 45 minutes after reperfusion, "60 minutes," 46 to 60 minutes after reperfusion, and "90 minutes," 61 to 90 minutes after reperfusion. 


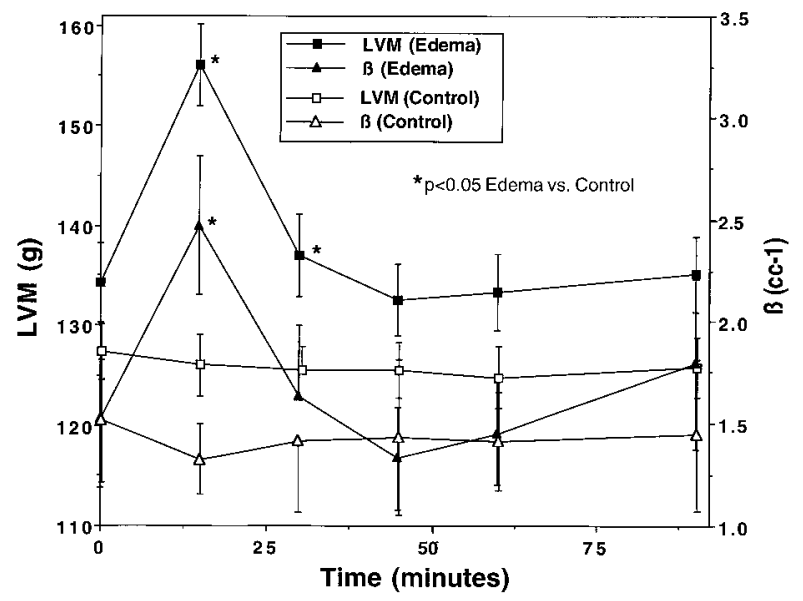

Fig. 3. Time course of mean LV mass $(L V M)$ calculated by 2 -DE and diastolic stiffness constant ( $\beta$ for edema $(n=$ $5)$ and control groups $(n=6)$. Statistically significant increases occur in $\beta$ and LV mass in the edema group at 15 minutes and then return toward normal.

As previously described, ${ }^{12}$ data digitized at $200 \mathrm{~Hz}$ were analyzed with the use of IGOR software (Wavemetrics, Inc., Lake Oswego, Ore.). Raw conductance was corrected for parallel conductance ${ }^{13}$ and $\alpha$. Corrected volume was then used in all calculations of systolic and diastolic function.

End-diastole was defined as the point on the pressure trace coinciding with the $\mathrm{R}$ wave on the electrocardiogram. Analysis of LV diastolic pressure-volume relationship consisted of exponential curve fitting of end-diastolic pressure (EDP) and volume (EDV) by the least squares method (equation 1). $\beta$ is the LV stiffness constant and $\alpha$ is the base constant.

$$
\mathrm{EDP}=\alpha \mathrm{ae} \mathrm{e}^{\beta \mathrm{EDV}}
$$

As suggested by Weiss, Frederiksen, and Weisfeldt, ${ }^{15}$ the diastolic relaxation constant, $\tau$, was determined from the slope, A, of the natural log of pressure versus time during isovolumic relaxation over $60 \mathrm{msec}$ after peak negative rate of pressure rise.

$$
\tau=-1 / \mathrm{A}
$$

End-systole was defined by the upper left-hand corner of the pressure-volume loop. ${ }^{16}$ The end-systolic pressure-volume relation defined maximum systolic elastance. ${ }^{16}$ Stroke work was determined for each cardiac cycle by calculating the area within the pressure-volume loop. Preload recruitable stroke work (PRSW) was defined by the relationship between stroke work and end-diastolic volume. ${ }^{17}$ The slopes and volume intercepts were defined by linear regression. Volume intercepts ( $\mathrm{x}$-axis intercepts) were calculated at the point where the fitted linear regression line had a value of zero.

Echo techniques, previously described, ${ }^{12}$ measured LV wall volume as the numeric difference of the epicardial

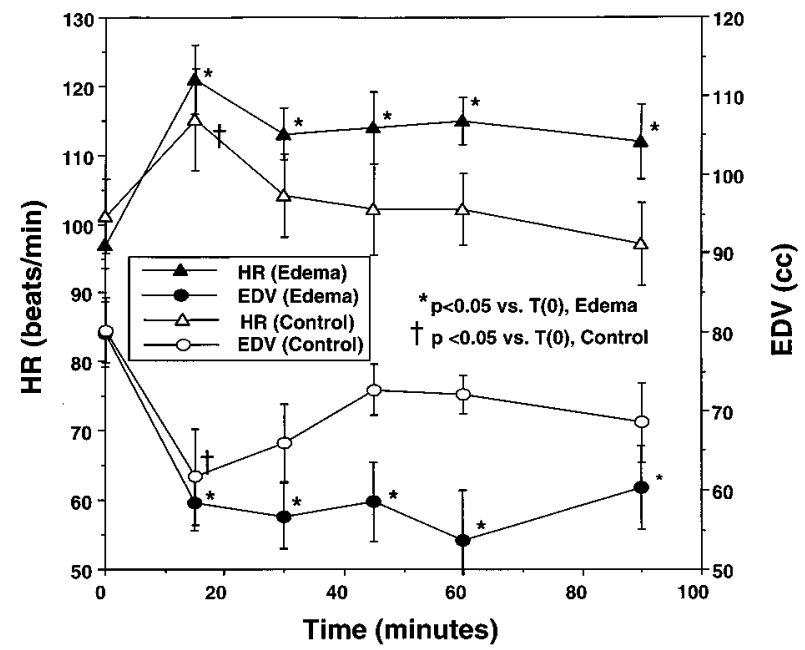

Fig. 4. Time course of heart rate $(H R)$ and end-diastolic volume $(E D V)$ for edema $(n=5)$ and control groups $(n=$ $6)$. Heart rate is increased significantly and end-diastolic volume is decreased significantly at the 15 -minute time point.

and endocardial shells, multiplied by myocardial specific gravity $(1.05 \mathrm{gm} / \mathrm{ml})$ to determine LV mass.

Myocardial water content (MWC) was calculated from equation 3:

$\operatorname{MWC}(\%)=([$ Wet heart weight -

Dry heart weight $] /[$ Wet heart weight] $) \times 100$

Statistical analysis. For comparisons between groups, LV mass, diastolic properties $(\alpha, \beta, \tau)$, systolic properties (systolic elastance and PRSW) were compared by repeated-measures analysis of variance (ANOVA). Similarly, conductance calibration before and after perfusion were compared by repeated-measures ANOVA. Post hoc comparisons were performed with the use of Tukey's procedure. Postmortem LV weight, LV myocardial water content, hematocrit value, perfusion pressure, and coronary perfusion time were compared by means of unpaired Student's $t$ test.

\section{Results}

Fig. 1 illustrates a representative pressure-conductance tracing during vena caval occlusion. Raw conductance was converted to LV volume by means of calibration routines based on injection of hypertonic saline solution. Fig. 2 illustrates effects of edema on the LV diastolic pressure-volume relationship during a representative experiment; the data shift upward and to the left in the edema group during reperfusion, reflecting increased diastolic stiffness.

Fig. 3 presents the time course of average LV mass, as determined by $2-\mathrm{DE}$ and $\beta$. LV mass and $\beta$ 
Table I. Systolic indices of $L V$

\begin{tabular}{|c|c|c|c|c|c|c|}
\hline & \multicolumn{6}{|c|}{ Time intervals (min) } \\
\hline & Pre & $3-15$ & $16-30$ & $31-45$ & $46-60$ & $61-90$ \\
\hline \multicolumn{7}{|c|}{ Cardiac output (ml/sec) } \\
\hline Edema $(n=5)$ & $4.6 \pm 0.3$ & $4.0 \pm 0.4$ & $4.8 \pm 0.6$ & $4.8 \pm 0.8$ & $4.8 \pm 0.9$ & $3.8 \pm 0.4$ \\
\hline Control $(n=6)$ & $4.6 \pm 0.3$ & $4.1 \pm 0.5$ & $4.0 \pm 0.4$ & $4.0 \pm 0.5$ & $3.7 \pm 0.4$ & $3.9 \pm 0.6$ \\
\hline \multicolumn{7}{|l|}{ PRSW (mm Hg) } \\
\hline Edema $(n=5)$ & $57.0 \pm 5.7$ & $55.2 \pm 4.2$ & $76.0 \pm 11.7$ & $83.8 \pm 11.7$ & $77.2 \pm 3.9$ & $77.8 \pm 10.2$ \\
\hline Control $(n=6)$ & $49.5 \pm 2.1$ & $61.1 \pm 6.7$ & $59.6 \pm 7.7$ & $63.6 \pm 7.0$ & $60.4 \pm 4.2$ & $59.1 \pm 7.7$ \\
\hline \multicolumn{7}{|l|}{ Es $(\mathrm{mm} \mathrm{Hg} / \mathrm{ml})$} \\
\hline Edema $(n=5)$ & $1.5 \pm 0.2$ & $2.2 \pm 0.5$ & $2.1 \pm 0.3$ & $1.9 \pm 0.4$ & $3.0 \pm 0.7$ & $2.8 \pm 0.6$ \\
\hline Control $(n=6)$ & $1.3 \pm 0.1$ & $3.1 \pm 0.4$ & $2.9 \pm 0.3$ & $3.0 \pm 0.4$ & $3.6 \pm 0.5$ & $2.5 \pm 0.3$ \\
\hline
\end{tabular}

Data are reported as mean \pm standard error of the mean. PRSW, Preload recruitable stroke work; Es, systolic elastance.

Table II. Mean perfusion data

\begin{tabular}{lccc}
\hline & $\begin{array}{c}\text { Control } \\
(n=6)\end{array}$ & $\begin{array}{l}\text { Edema } \\
(n=5)\end{array}$ & $p$ Value \\
\hline $\begin{array}{c}\text { Hematocrit value of } \\
\text { perfusate (\%) }\end{array}$ & $28 \pm 0.8$ & $9.8 \pm 0.8$ & $<0.01$ \\
Perfusion volume (ml) & $500 \pm 0.0$ & $500 \pm 0.0$ & 1.00 \\
$\begin{array}{l}\text { Perfusion pressure (mm Hg) } \\
\text { Duration of perfusion (sec) }\end{array}$ & $57.8 \pm 0.8$ & $57.4 \pm 0.9$ & 0.865 \\
& $50.7 \pm 3.7$ & $57.0 \pm 4.2$ & 0.170
\end{tabular}

Data are reported as mean \pm standard error of the mean. NS, Not significant.

increased significantly in the edema group compared with the control group in the first 15-minute interval after coronary perfusion, returning to control values by the 45 -minute time interval. There was no significant difference between preperfusion LV mass and postmortem weight. Myocardial water content was not different between the groups at the end of the experiment. Testing for subjective planimetry errors was done with a blinded second observer, as previously described. ${ }^{1,6,18,19}$ No intraobserver differences were noted. Postmortem LV weight correlated well with 2-DE LV mass.

The base constant, $\alpha$, and isovolumic relaxation constant, $\tau$, did not change significantly in the edema group as compared with the control group. Enddiastolic volume was not different between groups. However, within groups, end-diastolic volume significantly decreased from baseline in the 30-minute time interval in the control group, but remained lower in the edema group ( $p<0.05$, ANOVA). Heart rate did not differ between groups, but within each group it was significantly higher in the first 15-minute interval after removal of the aortic crossclamp, returning to baseline in the 30-minute interval in the control group only (Fig. 4).

Table I presents indices for systolic function. Measures of contractility, end-systolic pressure-vol- ume relationship and PRSW, were not statistically different between groups (repeated-measures ANOVA). Cardiac output decreased after reperfusion in both groups, but the change was not statistically significant.

Average perfusion hematocrit value, time of perfusion, and perfusion pressure are presented in Table II. There was no difference in perfusion pressure or time of perfusion between the two groups.

\section{Discussion}

The present experiment was designed from its inception to control extraneous influences on cardiac function and edema. The data demonstrate increased LV mass and impaired diastolic filling after coronary perfusion with hemodiluted blood. The LV end-diastolic pressure-volume relation shifts upward and leftward, indicating decreased LV compliance and increased chamber stiffness. Fortyfive minutes after whole blood reperfusion, LV mass and compliance return to control values. These results for the normal pig heart define the transient effects of hemodilution on LV geometry and diastolic properties and the minimum recovery time in the absence of specific treatment.

Many related observations have been reported. In isolated, hypothermic pig hearts, Hsu and cowork$\mathrm{ers}^{20}$ demonstrated that hyposmolar coronary perfusion increased both heart weight (by 30\%) and myocardial water content (by 6.5\%), consonant with simple models for fluid distribution. ${ }^{2}$ Foglia, Steed, and Follette ${ }^{7}$ demonstrated that hypoosmotic cardioplegia increased LV myocardial water content in a canine model undergoing $\mathrm{CPB}$. Others reported a $16 \%$ increase in LV mass with hemodilution ${ }^{18,19}$ in dogs undergoing $\mathrm{CPB}$; myocardial water content increased proportionately. A $22 \%$ increase in LV 
mass was also observed in dogs after 45 minutes of ischemic arrest on CPB. ${ }^{6}$ These observations and additional evidence support the view that increased LV mass in the present study reflects myocardial edema.

Several sources of error warrant discussion. Increased LV mass and LV wall thickness are interpreted in the present study to indicate myocardial edema. An alternate explanation for increased wall thickness is reactive hyperemia. ${ }^{21}$ We believe reactive hyperemia did not occur, because no increase in wall thickness occurred in a previous study ${ }^{12}$ after 1 minute of ischemic ventricular fibrillation and reperfusion. Hyperemia is even less likely in the present study, because fibrillation was avoided. We also argue that the hematocrit value of the perfusate was not a factor, because a hematocrit value of $9 \%$ is said to minimally impair myocardial oxygen delivery. $^{4,5}$ Any myocardial ischemia and hyperemia resulting from 1 minute of perfusion at this hematocrit value should have been too short-lived to have affected the results. The degree of hemodilution was based on a prior study. ${ }^{22}$

Conductance in the open chest is affected by metallic objects, temperature, right ventricular volume, lung volume, and size of the pericardial contact footprint. ${ }^{23}$ These can alter parallel conductance or the slope constant, or both. Frequent calibration was used to compensate for this. Since we found the calibration constants extremely stable, myocardial edema apparently does not affect conductance calibration. A tendency for parallel conductance to increase during reperfusion in our study could reflect changes in right ventricular volume, lung volume, or temperature. ${ }^{23}$

Conductance was useful for measuring instantaneous LV volume in this experiment, particularly because edema causes artifacts in other measuring techniques $^{1,24}$ and because laborious calculations are required to extend quantitative 2-DE throughout the cardiac cycle. For these reasons, 2-DE was used to measure LV mass, whereas conductance was used to measure LV volume. Conductance calibration based on 2-DE has been described. ${ }^{14}$

The last error source to be discussed is the use of exponential curve fitting for analysis of diastolic properties. This can be problematic at low filling pressures, where the curves are relatively flat and may even have a reversed sigmoidal shape. ${ }^{20}$ Our approach is simplistic and could be inaccurate at pressures approaching zero. However, Fig. 2 suggests that exponential curve fitting was reasonable for the present data set. The terminology for base and exponential constants has also been confusing; $\beta$ has been used for the base or exponential constant by different authors.

Effects of edema on systolic properties are not well understood. Although edema could depress contractile function, ${ }^{25,} 26$ systolic performance has not been impaired by edema in several prior studies. $^{3,6}$ Our data reveal no change in systolic elastance or PRSW after the first 5 minutes of reperfusion. Cardiac output in both groups decreased transiently and insignificantly after reperfusion, possibly reflecting changes in preload. The assessment of contractility may have been affected by the use of inotropic agents immediately after crossclamping and by systemic reflexes, which were not blocked.

Many factors modifying edema were not explored in this study. These include ischemic injury, which affects membrane properties and ion pumps, and $\mathrm{CPB}$, which releases cytokines and causes leaky membranes. Additional important factors are congestive heart failure, hypothermia, drug reactions, hypoproteinemia, sepsis, and transplant rejection. The purpose of the present study was not to describe myocardial edema in all its forms, but rather to define recovery from myocardial edema in its simplest form.

In summary, using a new model, myocardial edema was found to increase ventricular stiffness and wall volume. These changes resolved after 45 minutes of whole blood reperfusion. This model may facilitate testing cardioplegic agents and treatment for myocardial edema.

We gratefully acknowledge the technical assistance of Natalya Chalik and Michael J. Sardo.

REFERENCES

1. Haasler GB, Rodigas PC, Collins RH, Wei J, Meyer FJ, Spotnitz AJ, et al. Two-dimensional echocardiography in dogs: variation of left ventricular mass, geometry, volume, and ejection fraction on cardiopulmonary bypass. J Thorac Cardiovasc Surg 1985;90:430-40.

2. Spotnitz HM, Hsu DT. Myocardial edema: importance in the study of left ventricular function. Adv Card Surg 1994;5:1-25.

3. Cross CE, Rieben PA, Salisbury PF. Influence of coronary perfusion and myocardial edema on pressure-volume diagram of left ventricle. Am J Physiol 1961;201:102-8.

4. Laks H, Standeven J, Blair O, Hahn J, Jellinek M, Willman VL. The effects of cardiopulmonary bypass with crystalloid and colloid hemodilution on myocardial extravascular water. J Thorac Cardiovasc Surg 1977;73:129-38.

5. Geffin GA, Vasu MA, O'Keefe D, Pennington DG, Erdmann $\mathrm{AJ}$, Lowenstein E, et al. Ventricular performance and myo- 
cardial water content during hemodilution in dogs. Am J Physiol 1978;235:767-75.

6. Lazar HL, Hassler GB, Collins RH, Dubroff JM, Meisner J, Spotnitz HM. Compliance, mass, and shape of the canine left ventricle after global ischemia analyzed with two-dimensional echocardiography. J Surg Res 1985;39:199-208.

7. Foglia RP, Steed DL, Follette DM. Iatrogenic myocardial edema with potassium cardioplegia. J Thorac Cardiovasc Surg 1979;78:217-22.

8. Foglia RP, Lazar HL, Steed DL, Follette DM, Manganaro AJ, Deland E, et al. Iatrogenic myocardial edema with crystalloid primes: effects on left ventricular compliance, performance, and perfusion. Surg Forum 1978;29:312-5.

9. Vogel WM, Cerel AW, Apstein CS. Post-ischemic cardiac chamber stiffness and coronary vasomotion: the role of edema and effects of dextran. J Mol Cell Cardiol 1986;18: 1207-18.

10. Schaff HV, Gott VL, Goldman RA, Frederiksen JW, Flaherty JT. Mechanism of elevated left ventricular end-diastolic pressure after ischemic arrest and reperfusion. Am J Physiol 1981;240:H300-7.

11. Takoudes TG, Amirhamzeh MMR, Hsu DT, Wise BR, Odeh SO, Spotnitz HM. Time course of resolution of perfusioninduced myocardial edema in the rat heart. J Surg Res 1994;57:641-6.

12. Amirhamzeh MMR, Dean DA, Jia C-X, et al. Iatrogenic myocardial edema: increased diastolic compliance and time course to resolution in vivo. Ann Thorac Surg 1996;62:737-43.

13. Burkhoff D, Van der Valde ET, Kass D, Baan J, Maughan WL, Sagawa K. Accuracy of volume measurement by conductance catheter in isolated, ejecting canine hearts. Circulation 1985;72:440-7.

14. Cabreriza SE, Amirhamzeh MMR, Jia C-X, Spotnitz HM. Conductance-echocardiography correlations during changes in left ventricular volume. ASAIO J 1995;41:M669-73.

15. Weiss JL, Frederiksen JW, Weisfeldt ML. Hemodynamic determinants of the time-course of fall in the canine left ventricular pressure. J Clin Invest 1976;58:751-60.

16. Sagawa K. The ventricular pressure-volume diagram revisited. Circ Res 1978;43:677-86.
17. Glower DD, Spratt JA, Snow ND, Kabas JS, Davis JW, Olsen $\mathrm{CO}$, et al. Linearity of the Frank-Starling relationship in the intact heart: the concept of preload recruitable stroke work. Circulation 1985;71:994-1009.

18. Collins RH, Haasler GB, Krug JH, Colt C, Spotnitz AJ, Martin EC, Spotnitz HM. Canine left ventricular volume and mass during thoracotomy by two-dimensional echocardiography. J Surg Res 1982;33:294-304.

19. Hassler HB, Rodigas PC, Spotnitz HM. The absence of temperature effects on end-diastolic pressure-volume relations in the canine left ventricle determined by two-dimensional echocardiography. J Thorac Cardiovasc Surg 1982;83:878-90.

20. Hsu DT, Weng Z-C, Nicolosi AC, Detwiler PW, Sciacca R, Spotnitz HM. Quantitative effects of myocardial edema on the left ventricular pressure volume relation in the isolated stored pig heart. J Thorac Cardiovasc Surg 1993;106: 651-7.

21. Wexler LF, Grice WN, Huntington M, Plehn J, Apstein CS. Coronary hypertension and diastolic compliance in isolated rabbit hearts. Hypertension 1989;13:598-606.

22. Dean DA, Jia C-X, Cabreriza SE, Soto PF, Rabkin DG, Sardo $\mathrm{MJ}$, et al. Retrograde coronary perfusion: effects on iatrogenic edema and diastolic properties. Ann Thorac Surg 1998;65:44953.

23. Cabreriza SE, Dean DA, Jia C-X, Dickstein ML, Spotnitz HM. Electrical isolation of the heart: stabilizing parallel conductance for left ventricular volume measurement. ASAIO J 1997;43:M509-14.

24. Spotnitz WD, Clark MB, Rosenblum HM, Lazar HL, Haasler GB, Collins RH, et al. Effect of cardiopulmonary bypass and global ischemic on human and canine left ventricular mass: evidence for interspecies differences. Surgery 1984;96:230-8.

25. Godt RE, Maughan DW. Influence of osmotic compression on calcium activation and tension in skinned fibers of the rabbit. Pflugers Arch 1981;391:334-7.

26. Zhao M, Sonnenblick EH, Zang H, Eng C. Increase in myofilament separation in the "stunned" myocardium. J Mol Cell Cardiol 1992;24:269-76. 